\title{
Lie Elements and Knuth Relations
}

\author{
Manfred Schocker* \\ LaCIM, UQAM \\ CP 8888, succ. Centre-Ville \\ Montréal (Québec), H3C 3P8 \\ Canada \\ E-mail: mschock@math.uqam.ca
}

October 24, 2018

\begin{abstract}
A coplactic class in the symmetric group $\mathcal{S}_{n}$ consists of all permutations in $\mathcal{S}_{n}$ with a given Schensted $Q$-symbol, and may be described in terms of local relations introduced by Knuth. Any Lie element in the group algebra of $\mathcal{S}_{n}$ which is constant on coplactic classes is already constant on descent classes. As a consequence, the intersection of the Lie convolution algebra introduced by Patras and Reutenauer and the coplactic algebra introduced by Poirier and Reutenauer is the Solomon descent algebra.
\end{abstract}

MSC 2000: 17B01*, 05E10, 20C30, 16W30

\section{Introduction}

In 1995, Malvenuto and Reutenauer introduced the structure of a graded Hopf algebra on the direct sum

$$
\mathcal{P}=\bigoplus_{n \geq 0} \mathbb{Z} \mathcal{S}_{n}
$$

of all symmetric group algebras $\mathbb{Z} \mathcal{S}_{n}$ over the ring $\mathbb{Z}$ of integers ( MR95). Apart from this convolution algebra of permutations $\mathcal{P}$ itself (AS, DHT)

${ }^{*}$ supported by the Research Chairs of Canada 
several sub-algebras of $\mathcal{P}$ turned out to be of particular algebraic and combinatorial interest and have been studied intensively; for instance, the Rahmenalgebra ([J̈̈199]), the Hopf algebra of the planar binary trees ( LR98, Cha00),

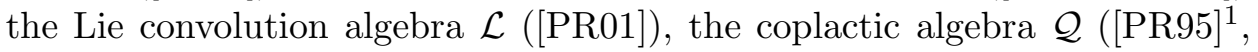
[BS]), and the Solomon descent algebra $\mathcal{D}$ (Sol76, GR89, Reu93, MR95, $\mathrm{GKL}^{+} 95$, BL96, JR01).

Here, the relation between the algebras $\mathcal{L}$ and $\mathcal{Q}$ shall be investigated. The latter is defined combinatorially as the linear span of the sums of permutations with given Schensted $Q$-symbol ([Sch61]), or, equivalently, of the sums of equivalence classes arising from the coplactic relations in $\mathcal{S}_{n}, n \geq 0$, introduced by Knuth $([\mathrm{Knu} 70])$. The Lie convolution algebra $\mathcal{L}$ is generated (as an algebra) by all Lie elements in $\mathcal{P}$. Both $\mathcal{L}$ and $\mathcal{Q}$ contain $\mathcal{D}$. Combinatorial descriptions of the algebras $\mathcal{D}$ and $\mathcal{Q}$, and the set of Lie elements in $\mathcal{P}$, follow in Section 2. The main goal of this paper is to show

Theorem 1. $\mathcal{L} \cap \mathcal{Q}=\mathcal{D}$.

This result (once more) points out the exceptional role played by the Solomon algebra. The proof is given in Section 2, and is essentially based on the fact that any Lie element in $\mathcal{P}$ which is constant on coplactic classes is already contained in $\mathcal{D}$ (see Section 3 ), which is combinatorially interesting for its own sake.

One might be tempted to conjecture that a lack of co-commutativity of $\mathcal{Q}$ is the deeper reason for Theorem $\mathbb{1}$, since $\mathcal{L}$ is - at least in comparison to $\mathcal{D}$ - a "large" co-commutative sub-algebra of $\mathcal{P}$; but this is false. The domain of co-commutativity of $\mathcal{Q}$ strictly contains $\mathcal{D}$. Some comments concerning this can be found at the end of Section 3 .

\section{Descent, Coplactic, and Lie Relations}

In this section, combinatorial descriptions of the algebras $\mathcal{D}$ and $\mathcal{Q}$, and of the Lie elements in $\mathcal{P}$, are recalled briefly; and a proof of Theorem 1 is given.

Let $\mathbb{N}$ (respectively, $\mathbb{N}_{0}$ ) be the set of positive (respectively, nonnegative) integers and set

$$
\underline{n}:=\{i \in \mathbb{N} \mid i \leq n\}
$$

\footnotetext{
${ }^{1}$ The algebra $(\mathbb{Z} C, *, \delta)$ introduced in PR95 is the dual algebra of the algebra $\mathcal{Q}$ considered here (see PR95, Théorème 3.4]).
} 
for all integers $n$. For any $\pi \in \mathcal{S}_{n}$, $\operatorname{Des}(\pi):=\{i \in \underline{n-1} \mid i \pi>(i+1) \pi\}$ is the descent set of $\pi$. The Solomon descent algebra $\mathcal{D}$ is the linear span of the sums $\sum_{\substack{\pi \in \mathcal{S}_{n} \\ \operatorname{Des}(\pi)=D}} \pi$, where $n \in \mathbb{N}_{0}$ and $D \subseteq \underline{n-1}$. Due to Malvenuto and Reutenauer, $\mathcal{D}$ is a Hopf sub-algebra of $\mathcal{P}$ ([MR95]). We mention that the homogeneous component $\mathcal{D}_{n}=\mathcal{D} \cap \mathbb{Z} \mathcal{S}_{n}$ is a sub-algebra of $\mathbb{Z} \mathcal{S}_{n}$, due to a remarkable result of Solomon (Sol76]), although this is not of relevance here.

Let $\mathbb{N}^{*}$ be a free monoid over the alphabet $\mathbb{N}$ and denote by $\varnothing$ the empty word in $\mathbb{N}^{*}$. The mapping $\pi \mapsto(1 \pi) \ldots(n \pi)$ extends to a linear embedding of $\mathbb{Z} \mathcal{S}_{n}$ into the semi-group algebra $\mathbb{Z} \mathbb{N}^{*}$. As is convenient for our purposes, elements of $\mathbb{Z} \mathcal{S}_{n}$ will be identified with the corresponding elements of $\mathbb{Z} \mathbb{N}^{*}$. Furthermore, products $\sigma \nu$ of permutations $\sigma, \nu \in \mathcal{S}_{n}$ are to be read from left to right: first $\sigma$, then $\pi$.

The following combinatorial characterization of $\mathcal{D}_{n}$ was given in [BL93, 4.2].

Proposition 2.1 (Descent Relations). Let $\varphi=\sum_{\nu \in \mathcal{S}_{n}} k_{\nu} \nu \in \mathbb{Z} \mathcal{S}_{n}$, then $\varphi \in \mathcal{D}_{n}$ if and only if

$$
k_{u a w(a+1) v}=k_{u(a+1) w a v}
$$

for all $a \in \underline{n-1}, u, v, w \in \mathbb{N}^{*}$ such that $\nu=u a w(a+1) v \in \mathcal{S}_{n}$ and $w \neq \varnothing$.

Let $Q(\pi)$ denote the Schensted $Q$-symbol of $\pi$, for all $\pi \in \mathcal{S}_{n}$ ( Sch61] $)$, then the set of all $\sigma \in \mathcal{S}_{n}$ such that $Q(\pi)=Q(\sigma)$ is a coplactic class in $\mathcal{S}_{n}$ ?. The coplactic algebra $\mathcal{Q}$ is the linear span of all sums of coplactic classes in $\mathcal{P}$ :

$$
\mathcal{Q}=\left\langle\left\{\sum_{Q(\sigma)=Q(\pi)} \sigma \mid \pi \in \mathcal{S}_{n}, n \in \mathbb{N}_{0}\right\}\right\rangle_{\mathbb{Z}} .
$$

Accordingly, each element $\varphi \in \mathcal{Q}$ is called coplactic. Due to Poirier and Reutenauer, $\mathcal{Q}$ is a Hopf sub-algebra of $\mathcal{P}$ ( terization of $\mathcal{Q}_{n}:=\mathcal{Q} \cap \mathbb{Z} \mathcal{S}_{n}$ is due to Knuth ([Knu70]).

Proposition 2.2 (Coplactic Relations). Let $\varphi=\sum_{\nu \in \mathcal{S}_{n}} k_{\nu} \nu \in \mathbb{Z} \mathcal{S}_{n}$, then $\varphi \in \mathcal{Q}_{n}$ if and only if

$$
k_{u a w(a+1) v}=k_{u(a+1) w a v}
$$

for all $a \in \underline{n-1}, u, v, w \in \mathbb{N}^{*}$ such that $\nu=u a w(a+1) v \in \mathcal{S}_{n}$ and $w$ contains the letter $a-1$ or the letter $a+2$.

\footnotetext{
${ }^{2}$ Due to Schützenberger $(\sqrt{\operatorname{Sch} 63}), P(\pi)=Q\left(\pi^{-1}\right)$ is the Schensted $P$-symbol of $\pi$; and the equivalence arising from equality of $P$-symbols leads to the plactic monoid ([LS81]). This is the reason why the word coplactic is used here.
} 
Combining Propositions 2.1 and 2.2 implies, in particular, $\mathcal{D} \subseteq \mathcal{Q}$.

Let

$$
\omega_{n}=\sum_{\pi}(-1)^{1 \pi^{-1}-1} \pi \in \mathbb{Z} \mathcal{S}_{n},
$$

where the sum is taken over all valley permutations $\pi \in \mathcal{S}_{n}$, which are defined by the property $1 \pi>\cdots>(k-1) \pi>k \pi<(k+1) \pi<\cdots<n \pi$, where $k:=1 \pi^{-1}$. The element $\omega_{n}$ projects $\mathbb{Z} \mathcal{S}_{n}$ onto the multi-linear part of the free Lie algebra, by left multiplication (Dyn47, Spe48, Wev49, see BL93]). Accordingly,

$$
\operatorname{Lie}_{n}:=\omega_{n} \mathbb{Z} \mathcal{S}_{n}
$$

is the set of Lie elements in $\mathbb{Z} \mathcal{S}_{n}$ for all $n \in \mathbb{N}_{0}$. Each $\varphi \in$ Lie $:=\bigoplus_{n>0}$ Lie $_{n}$ is a primitive element of the Hopf algebra $\mathcal{P}([\overline{\text { PR01 }}])$. The Lie convolution algebra $\mathcal{L}$ is the (co-commutative) Hopf sub-algebra of $\mathcal{P}$ generated by Lie; there is also the relation $\mathcal{D} \subseteq \mathcal{L}([$ PR01] $)$.

In view of a proof of Theorem 1 , consider the corresponding algebras $\mathcal{D}_{\mathbb{Q}}$, $\mathcal{L}_{\mathbb{Q}}, \mathcal{Q}_{\mathbb{Q}}$, and $\mathcal{P}_{\mathbb{Q}}$ over the field $\mathbb{Q}$ of rational numbers, then $\mathcal{D}_{\mathbb{Q}}$ is contained in $\mathcal{L}_{\mathbb{Q}} \cap \mathcal{Q}_{\mathbb{Q}}$; the latter is a co-commutative Hopf sub-algebra of $\mathcal{P}_{\mathbb{Q}}$, hence generated by its primitive elements, due to Milnor and Moore (MM65). But each primitive element in $\mathcal{L}_{\mathbb{Q}} \cap \mathcal{Q}_{\mathbb{Q}}$ is, in particular, a primitive element in $\mathcal{L}_{\mathbb{Q}}$ and therefore (up to a rational factor) contained in Lie. In Section 3 , it will be shown that any coplactic Lie element $\varphi \in \operatorname{Lie} \cap \mathcal{Q}$ is contained in $\mathcal{D}$ (Theorem 2). This implies $\mathcal{L}_{\mathbb{Q}} \cap \mathcal{Q}_{\mathbb{Q}} \subseteq \mathcal{D}_{\mathbb{Q}}$. Observing that $\mathcal{D}_{\mathbb{Q}} \cap \mathcal{P}=\mathcal{D}$, completes the proof of Theorem 1 .

A combinatorial characterization of the set $\mathrm{Lie}_{n}$ follows. Let $u ш v$ denote the usual shuffle product of $u=u_{1} \ldots u_{k}, v=v_{1} \ldots v_{m} \in \mathbb{N}^{*}$, that is

$$
u ш v=\sum_{w} w
$$

where the sum ranges over all $w=w_{1} \ldots w_{m+k} \in \mathbb{N}^{*}$ such that $u=$ $w_{i_{1}} \ldots w_{i_{k}}$ and $v=w_{j_{1}} \ldots w_{j_{m}}$ for suitably chosen indices $i_{1}<\cdots<i_{k}$, $j_{1}<\cdots<j_{m}$ such that $\underline{k+m}=\left\{i_{1}, \ldots, i_{k}, j_{1}, \ldots, j_{m}\right\}$. Furthermore, set

$$
\bar{u}:=u_{k} \ldots u_{1}
$$

and denote by $\ell(u):=k$ the length of $u$.

Proposition 2.3. Let $n \in \mathbb{N}$ and $a \in \underline{n}$, then $\left\{\omega_{n} \sigma \mid \sigma \in \mathcal{S}_{n}, 1 \sigma=a\right\}$ is a linear basis of $\mathrm{Lie}_{n}$. 
Furthermore, for any choice of coefficients $c_{\sigma} \in \mathbb{Z}\left(\sigma \in \mathcal{S}_{n}, 1 \sigma=a\right)$, the coefficient of $\nu=u a v \in \mathcal{S}_{n}$ in $\omega_{n} \sum_{1 \sigma=a} c_{\sigma} \sigma$ is

$$
(-1)^{\ell(u)} c_{a(\bar{u} \boldsymbol{\omega v})},
$$

where $\pi \mapsto c_{\pi}$ has been extended to $\mathbb{Z} \mathcal{S}_{n}$ linearly. In particular, the coefficient of $\sigma \in \mathcal{S}_{n}$ is $c_{\sigma}$ whenever $1 \sigma=a$.

This result is seemingly folklore; a proof follows for the reader's convenience.

Proof. Let $\nu=u a v \in \mathcal{S}_{n}$ and $\sigma=a x_{2} \ldots x_{n} \in \mathcal{S}_{n}$, then the coefficient of $\nu$ in $\omega_{n} \sigma$ is non-zero if and only if there is a valley permutation $\pi \in \mathcal{S}_{n}$ such that

$$
u a v=\nu=\pi \sigma=x_{1 \pi} \ldots x_{(k-1) \pi} a x_{(k+1) \pi} \ldots x_{n \pi},
$$

where $k:=1 \pi^{-1}$; that is, $u=x_{1 \pi} \ldots x_{(k-1) \pi}$ and $v=x_{(k+1) \pi} \ldots x_{n \pi}$. Since $1 \pi>\cdots>(k-1) \pi$ and $(k+1) \pi<\cdots<n \pi$, this is equivalent to saying that $x_{2} \ldots x_{n}$ is a summand in the shuffle product of $\bar{u}$ and $v$; in this case, the coefficient of $\nu$ in $\omega_{n} \sigma$ is $(-1)^{1 \pi^{-1}-1}=(-1)^{\ell(u)}$. This proves (11). Since

$$
\operatorname{dim} \operatorname{Lie}_{n}=(n-1) !=\#\left\{\omega_{n} \sigma \mid \sigma \in \mathcal{S}_{n}, 1 \sigma=a\right\}
$$

and the coefficient of $\tilde{\sigma}=a v \in \mathcal{S}_{n}$ in $\omega_{n} \sum_{1 \sigma=a} c_{\sigma} \sigma$ is $c_{\tilde{\sigma}}$, the basis property follows.

Corollary 2.4 (Lie relations). Let $\varphi=\sum_{\nu \in \mathcal{S}_{n}} k_{\nu} \nu \in \mathbb{Z} \mathcal{S}_{n}$, then $\varphi \in \mathrm{Lie}_{n}$ if and only if

$$
k_{u a v}=(-1)^{\ell(u)} k_{a(\bar{u} ш v)}
$$

for all $a \in \underline{n}, u, v \in \mathbb{N}^{*}$ such that $\nu=$ uav $\in \mathcal{S}_{n}$.

Proof. Let $\varphi \in \mathrm{Lie}_{n}$ and $a \in \underline{n}$, then there are coefficients $c_{\sigma} \in \mathbb{Z}\left(\sigma \in \mathcal{S}_{n}\right.$, $1 \sigma=a)$ such that $\varphi=\omega_{n} \sum_{1 \sigma=a} c_{\sigma} \sigma$, by Proposition 2.3, and

$$
k_{u a v}=(-1)^{\ell(u)} c_{a(\bar{u} \omega v)}=(-1)^{\ell(u)} k_{a(\bar{u} ш v)},
$$

by (11). Conversely, (2) implies $\varphi=\omega_{n} \sum_{1 \sigma=a} k_{\sigma} \sigma \in \mathrm{Lie}_{n}$, by (11) again.

Proposition 2.2 and Corollary 2.4 may be restated as follows. Let $T^{\perp}$ be the space orthogonal to $T$ with respect to the scalar product on $\mathbb{Z} \mathcal{S}_{n}$ turning $\mathcal{S}_{n}$ into an orthonormal basis, for all $T \subseteq \mathbb{Z} \mathcal{S}_{n}$. For all $c, d \in \mathbb{Z} \mathcal{S}_{n}$, write

$$
\left.c \equiv_{\mathrm{Q}} d \text { (respectively, } c \equiv_{\mathrm{L}} d, c \equiv \equiv_{\mathrm{LQ}} d\right) \text {, }
$$


if $c-d \in \mathcal{Q}_{n}^{\perp}$ (respectively, $\left.\in \operatorname{Lie}_{n}^{\perp}, \in\left(\operatorname{Lie}_{n} \cap \mathcal{Q}_{n}\right)^{\perp}\right)$. Now the necessity parts of Proposition 2.2 and Corollary 2.4 are

$$
u a w(a+1) v \equiv_{\mathrm{Q}} u(a+1) w a v
$$

for all $a \in \underline{n-1}, u, v, w \in \mathbb{N}^{*}$ such that $u a w(a+1) v \in \mathcal{S}_{n}$ and $w$ contains the letter $a-1$ or the letter $a+2$;

$$
u a v \equiv_{\mathrm{L}}(-1)^{\ell(u)} a(\bar{u} \omega v)
$$

for all $a \in \underline{n}, u, v \in \mathbb{N}^{*}$ such that $u a v \in \mathcal{S}_{n}$. For later use, note that applying (函) twice gives

$$
a u b v \equiv_{\mathrm{L}}(-1)^{n-1} \bar{v} b \bar{u} a \equiv_{\mathrm{L}}(-1)^{n-1+\ell(v)} b(v ш \bar{u} a)
$$

whenever $a, b \in \underline{n}$ and $u, v \in \mathbb{N}^{*}$ such that $a u b v \in \mathcal{S}_{n}$.

Remark. The space $\mathrm{Lie}_{n}^{\perp}$ is linearly generated by all non-trivial shuffles $u \omega v$, where $u, v \in \mathbb{N}^{*}$ such that $u v \in \mathcal{S}_{n}$ (see, for instance, Duc91). As a consequence of Corollary 2.4, for fixed $a \in \underline{n}$, the elements

$$
u a v-(-1)^{|u|} a(\bar{u} \boldsymbol{\omega} v),
$$

where $u, v \in \mathbb{N}^{*}$ such that uav $\in \mathcal{S}_{n}$ and $u \neq \varnothing$, constitute a linear basis of Lie $_{n}^{\perp}$. Another basis has been introduced by Duchamp (ibid.). This was pointed out to me by Christophe Reutenauer.

This section concludes with a helpful observation concerning the order reversing involution $\varrho_{n}=n(n-1) \ldots 1 \in \mathcal{S}_{n}$.

Proposition 2.5. $\varrho_{n} \mathrm{Lie}_{n}+\mathrm{Lie}_{n} \varrho_{n} \subseteq \mathrm{Lie}_{n}$, and $\varrho_{n} \mathcal{Q}_{n}+\mathcal{Q}_{n} \varrho_{n} \subseteq \mathcal{Q}_{n}$.

In particular, $\pi \equiv \equiv_{\mathrm{LQ}} \sigma$ implies $\pi \varrho_{n} \equiv \mathrm{LQ} \sigma \varrho_{n}$ and $\varrho_{n} \pi \equiv \mathrm{LQ}_{\mathrm{LQ}} \varrho_{n} \sigma$, for all $\pi, \sigma \in \mathcal{S}_{n}$.

Proof. First, $\varrho_{n} \omega_{n}=(-1)^{n-1} \omega_{n}$ yields $\varrho_{n} \mathrm{Lie}_{n} \subseteq \mathrm{Lie}_{n}$, while $\operatorname{Lie}_{n} \varrho_{n} \subseteq \mathrm{Lie}_{n}$ is obvious; and second, if $\sigma, \pi \in \mathcal{S}_{n}$ such that $\sigma \equiv_{\mathrm{Q}} \pi$, then $\sigma \varrho_{n} \equiv_{\mathrm{Q}} \pi \varrho_{n}$ and $\varrho_{n} \sigma \equiv_{\mathrm{Q}} \varrho_{n} \pi$, as is readily seen from Proposition 2.2. This implies $\varrho_{n} \mathcal{Q}_{n} \subseteq \mathcal{Q}_{n}$ and $\mathcal{Q}_{n} \varrho_{n} \subseteq \mathcal{Q}_{n}$.

In particular, it follows that $\varrho_{n}\left(\mathrm{Lie}_{n} \cap \mathcal{Q}_{n}\right)^{\perp}+\left(\mathrm{Lie}_{n} \cap \mathcal{Q}_{n}\right)^{\perp} \varrho_{n} \subseteq\left(\mathrm{Lie}_{n} \cap \mathcal{Q}_{n}\right)^{\perp}$, since $\varrho_{n}$ is an involution. 


\section{Coplactic Lie Elements}

The aim of this section is to show Lie $\cap \mathcal{Q} \subseteq \mathcal{D}$, which implies Theorem 1, as was mentioned in the previous section. Throughout, $n \in \mathbb{N}$ is fixed. Bearing in mind Proposition 2.1, it suffices to show that

$$
u a w(a+1) s \equiv \mathrm{LQ} u(a+1) \text { was }
$$

whenever $a \in \underline{n-1}, u, v, w \in \mathbb{N}^{*}$ such that $u a w(a+1) v \in \mathcal{S}_{n}$ and $w \neq \varnothing$.

The essential idea of the proof is illustrated by the following

Example. Let $\pi=15234, \sigma=25134 \in \mathcal{S}_{5}$, then $\pi$ and $\sigma$ are in descent, but not in coplactic relation. Applying (A) yields, however,

$$
\begin{aligned}
& \pi \quad \equiv_{\mathrm{L}} \quad-5(1 ш 234) \\
& =\quad-51234-52134-52314-52341 \\
& \equiv_{\mathrm{Q}} \quad-51234-52134-51324-51342 \\
& =-5(2 ш 134) \\
& \equiv{ }_{\mathrm{L}} \quad \sigma,
\end{aligned}
$$

hence $\pi \equiv{ }_{\mathrm{LQ}} \sigma$.

Some additional preparations are needed for the proof of (6). $v \in \mathbb{N}^{*}$ is called a sub-word of $w=w_{1} \ldots w_{m} \in \mathbb{N}^{*}$ if there exist $k \in \underline{m}$ and $1 \leq i_{1}<$ $\cdots<i_{k} \leq m$ such that $v=w_{i_{1}} \ldots w_{i_{k}}$. For instance, 23 is a sub-word of 52143.

For $a \in \underline{n-1}$, denote by $\tau_{a}=(a a+1)$ the transposition in $\mathcal{S}_{n}$ swapping $a$ and $a+1$. The word $v$ allows the a-switch in $\mathcal{S}_{n}$ if $\pi \equiv \mathrm{LQ} \pi \tau_{a}$ for all $\pi=\operatorname{uaw}(a+1) s \in \mathcal{S}_{n}$ such that $v$ is a sub-word of $w$. For instance, $v=a+2$ and $v=a-1$ allow the $a$-switch in $\mathcal{S}_{n}$, by (3). To save trouble, let it be said that, if $v$ contains a letter twice or a letter $b>n$ or $b \in\{a, a+1\}$, then $v$ allows the $a$-switch in $\mathcal{S}_{n}$; for in this case, there is no permutation $\pi=\operatorname{uaw}(a+1) s \in \mathcal{S}_{n}$ such that $v$ is a sub-word of $w$. Another way of stating (6) now is that $v \in \mathbb{N}^{*}$ allows the $a$-switch in $\mathcal{S}_{n}$ whenever $v \neq \varnothing$. The following three helpful observations will be applied frequently.

Proposition 3.1. Let $v \in \mathbb{N}^{*}$ such that $\pi \equiv \equiv_{\mathrm{LQ}} \pi \tau_{a}$ for all

$$
\pi=a w(a+1) s \in \mathcal{S}_{n}
$$

such that $v$ is a sub-word of $w$, then $v$ allows the a-switch in $\mathcal{S}_{n}$. 
Proof. Let $\pi=u a w(a+1) s \in \mathcal{S}_{n}$ such that $v$ is a sub-word of $w$, then

$$
\pi \equiv_{\mathrm{L}}(-1)^{\ell(u)} a(\bar{u} \boldsymbol{\omega} w(a+1) s),
$$

by (雨). Each summand in this shuffle product is of the form $a \hat{w}(a+1) \hat{s}$ such that $w$ (hence also $v$ ) is a sub-word of $\hat{w}$. It follows that

$$
\pi \equiv_{\mathrm{LQ}}(-1)^{\ell(u)}(a+1)(\bar{u} \boldsymbol{w} w a s) \equiv_{\mathrm{L}} u(a+1) \text { was },
$$

hence $v$ allows the $a$-switch in $\mathcal{S}_{n}$.

Proposition 3.2. Let $v=v_{1} \ldots v_{m} \in \mathbb{N}^{*}$ and assume that $v$ allows the a-switch in $\mathcal{S}_{n}$, then so does $\bar{v}$. Furthermore, if

$$
\tilde{v}:=\left(n+1-v_{1}\right) \ldots\left(n+1-v_{m}\right) \in \mathbb{N}^{*},
$$

then $\tilde{v}$ allows the $(n-a)$-switch in $\mathcal{S}_{n}$.

This is an immediate consequence of Proposition 2.5.

Proposition 3.3. Let $\pi \in \mathcal{S}_{n}$ and $\varphi_{0}, \ldots, \varphi_{m} \in \mathbb{Z} \mathcal{S}_{n}$ such that

(i) $\varphi_{0}=\pi$,

(ii) $\varphi_{i} \equiv_{\mathrm{L}} \varphi_{i+1}$, or $\varphi_{i} \equiv_{\mathrm{Q}} \varphi_{i+1}$ and $\varphi_{i} \tau_{a} \equiv_{\mathrm{Q}} \varphi_{i+1} \tau_{a}$, for all $i \in \underline{m-1} \cup\{0\}$,

(iii) $\varphi_{m} \equiv \mathrm{LQ} \varphi_{m} \tau_{a}$,

then $\pi \equiv \mathrm{LQ} \pi \tau_{a}$.

Proof. $\varphi \equiv_{\mathrm{L}} \psi$ implies $\varphi \tau_{a} \equiv_{\mathrm{L}} \psi \tau_{a}$ for all $\varphi, \psi \in \mathbb{Z} \mathcal{S}_{n}$, since $\operatorname{Lie}_{n} \tau_{a}=\mathrm{Lie}_{n}$. Combined with (ii), this implies $\varphi_{i} \tau_{a} \equiv \mathrm{LQ} \varphi_{i+1} \tau_{a}$ for all $i \in \underline{m-1} \cup\{0\}$, hence

$$
\pi=\varphi_{0} \equiv{ }_{\mathrm{LQ}} \varphi_{1} \cdots \equiv \equiv_{\mathrm{LQ}} \varphi_{m} \equiv \mathrm{LQ} \varphi_{m} \tau_{a} \equiv \mathrm{LQ} \cdots \equiv{ }_{\mathrm{LQ}} \varphi_{1} \tau_{a} \equiv{ }_{\mathrm{LQ}} \varphi_{0} \tau_{a}=\pi \tau_{a}
$$

by (i) and (iii).

We now show in four steps that each $v \in \mathbb{N}^{*} \backslash\{\varnothing\}$ allows the $a$-switch in $\mathcal{S}_{n}$.

Step 1. Let $a, k \in \underline{n-1}$ such that $k>a+1$, then $k(k+1)$ and $(k+1) k$ allow the a-switch in $\mathcal{S}_{n}$. 
Proof. Let $\pi=a w(a+1) s \in \mathcal{S}_{n}$ such that $k(k+1)$ or $(k+1) k$ is a sub-word of $w$. It suffices to prove $\pi \equiv_{\mathrm{Q}} \pi \tau_{a}$, by Proposition 3.1.

If $k=a+2$, then this follows from (3). Let $k \geq a+3$, and proceed by induction on $k$.

If $k-1$ occurs in $w$, then $\pi \equiv \mathrm{LQ} \pi \tau_{a}$, by induction. Let $k-1$ occur in $s$.

If $k=a+3$ and $\pi=a u_{1}(a+3) u_{2}(a+4) u_{3}(a+1) u_{4}(a+2) u_{5}$, then

$$
\pi \equiv_{\mathrm{Q}} a u_{1}(a+2) u_{2}(a+4) u_{3}(a+1) u_{4}(a+3) u_{5} .
$$

Applying Proposition 3.3, yields $\pi \equiv_{\mathrm{LQ}} \pi \tau_{a}$ in this case. In particular, $(a+3)(a+4)$ allows the $a$-switch in $\mathcal{S}_{n}$, hence also $(a+4)(a+3)$, by Proposition 3.2.

Now let $k>a+3$. If $k-2$ occurs in $w$, then there are $u_{i} \in \mathbb{N}^{*}(i \in \underline{5})$ such that either

$$
\begin{aligned}
\pi & =a u_{1} k u_{2}(k-2) u_{3}(a+1) u_{4}(k-1) u_{5} \\
& \equiv_{Q} a u_{1}(k-1) u_{2}(k-2) u_{3}(a+1) u_{4} k u_{5}=: \varphi
\end{aligned}
$$

or

$$
\begin{aligned}
\pi & =a u_{1}(k-2) u_{2} k u_{3}(a+1) u_{4}(k-1) u_{5} \\
& \equiv_{\mathrm{Q}} a u_{1}(k-1) u_{2} k u_{3}(a+1) u_{4}(k-2) u_{5}=: \varphi .
\end{aligned}
$$

In both cases, $\varphi \equiv_{\mathrm{LQ}} \varphi \tau_{a}$, by induction, hence $\pi \equiv_{\mathrm{LQ}} \pi \tau_{a}$, by Proposition 3.3 . Assume that $k-2$ occurs in $s$, then there are $u_{i} \in \mathbb{N}^{*}(i \in \underline{6})$ such that one of the following four cases holds.

case 1. $\pi=a u_{1}(k+1) u_{2} k u_{3}(a+1) u_{4}(k-2) u_{5}(k-1) u_{6}$, then $\pi \equiv_{\mathrm{Q}} \pi \tau_{k-1} \equiv_{\mathrm{Q}}$ $\pi \tau_{k-1} \tau_{k}$, and

$$
\pi \tau_{k-1} \tau_{k}=a u_{1} k u_{2}(k-1) u_{3}(a+1) u_{4}(k-2) u_{5}(k+1) u_{6} \equiv_{\mathrm{LQ}}\left(\pi \tau_{k-1} \tau_{k}\right) \tau_{a},
$$

by induction. Again Proposition 3.3 implies $\pi \equiv \mathrm{LQ} \pi \tau_{a}$.

case 2. $\pi=a u_{1} k u_{2}(k+1) u_{3}(a+1) u_{4}(k-1) u_{5}(k-2) u_{6}$, then put $m:=$ $\ell\left(u_{4}\right)+\ell\left(u_{5}\right)+\ell\left(u_{6}\right)+2$ and

$$
\varphi:=(-1)^{n-1+m}(a+1)\left(u_{4} k u_{5}(k-2) u_{6} ш \bar{u}_{3}(k+1) \bar{u}_{2}(k-1) \bar{u}_{1} a\right)
$$

to obtain $\pi \equiv_{\mathrm{Q}} a u_{1}(k-1) u_{2}(k+1) u_{3}(a+1) u_{4} k u_{5}(k-2) u_{6} \equiv_{\mathrm{L}} \varphi$, by (5). For all summands $\nu$ in $\varphi$ with $k$ to the left of $a$, that is $k \nu^{-1}<a \nu^{-1}, \nu \equiv \mathrm{LQ} \nu \tau_{a}$, 
by induction, while each of the summands $\nu$ with $k$ to the right of $a$ is of the form

$$
\nu=(a+1) v_{1}(k+1) v_{2}(k-1) v_{3} a v_{4} k v_{5} \equiv_{\mathrm{Q}} \nu \tau_{k},
$$

hence $\nu \equiv_{\mathrm{LQ}} \nu \tau_{a}$, by induction and Proposition 3.3. Putting both parts together yields $\varphi \equiv_{\mathrm{LQ}} \varphi \tau_{a}$, hence $\pi \equiv_{\mathrm{LQ}} \pi \tau_{a}$, by Proposition 3.3 .

case 3. $\pi=a u_{1}(k+1) u_{2} k u_{3}(a+1) u_{4}(k-1) u_{5}(k-2) u_{6}$, then putting $m:=\ell\left(u_{4}\right)+\ell\left(u_{5}\right)+\ell\left(u_{6}\right)+2$,

$$
\pi \equiv_{\mathrm{L}}(-1)^{n-1+m}(a+1)\left(u_{4}(k-1) u_{5}(k-2) u_{6} \omega \bar{u}_{3} k \bar{u}_{2}(k+1) \bar{u}_{1} a\right),
$$

by (5). For each of the summands, swapping $a$ and $a+1$ yields an LKequivalent permutation, since either $k-1$ stands to the left of $a$ and the induction hypothesis may be applied, or $k-1$ stands to the right of $a$ and case 2 may be applied. Thus $\pi \equiv_{\mathrm{LQ}} \pi \tau_{a}$, by Proposition 3.3 .

Combining cases 1 and 3 shows that $(k+1) k$ allows the $a$-switch in $\mathcal{S}_{n}$, hence also $k(k+1)$, by Proposition 3.2. This, in particular, yields the assertion in the remaining

case 4. $\pi=a u_{1} k u_{2}(k+1) u_{3}(a+1) u_{4}(k-2) u_{5}(k-1) u_{6}$.

For the proof of the second step, an auxiliary result is needed.

Proposition 3.4. Let $j, k, x \in \underline{n}, v_{1}, \ldots, v_{k+1}, w \in \mathbb{N}^{*}$ and set $x_{j}:=x+j$ for all $j \in \underline{k+1}$. If

$$
\pi:=v_{1} x_{1} v_{2} x_{2} \ldots v_{j-1} x_{j-1} v_{k+1} x_{k+1} v_{j} x_{j} v_{j+1} x_{j+1} \ldots v_{k} x_{k} \quad w
$$

is contained in $\mathcal{S}_{n}$, then

$$
\pi \equiv \equiv_{\mathrm{Q}} v_{1} x_{2} v_{2} x_{3} \ldots v_{j-1} x_{j} \quad v_{k+1} x_{j+1} v_{j} x_{1} v_{j+1} x_{j+2} \ldots v_{k} x_{k+1} w
$$

Proof. Let $u:=v_{1} x_{1} v_{2} x_{2} \ldots v_{j-1} x_{j-1}$ and $\hat{u}:=v_{j+1} x_{j+2} \ldots v_{k} x_{k+1} w$, then

$$
\begin{aligned}
& \pi=u v_{k+1} \underline{x_{k+1}} v_{j} x_{j} v_{j+1} x_{j+1} \ldots v_{k-1} x_{k-1} v_{k} \underline{x_{k}} w \\
& \equiv_{\mathrm{Q}} \quad u v_{k+1} \underline{x_{k}} \quad v_{j} x_{j} v_{j+1} x_{j+1} \ldots v_{k-1} \underline{x_{k-1}} v_{k} x_{k+1} w \\
& \equiv{ }_{Q} \quad \cdots \\
& \equiv_{\mathrm{Q}} \quad u v_{k+1} x_{j+1} v_{j} x_{j} v_{j+1} x_{j+2} \ldots v_{k-1} x_{k} \quad v_{k} x_{k+1} w \\
& =v_{1} x_{1} v_{2} x_{2} \ldots v_{j-2} x_{j-2} v_{j-1} \underline{x_{j-1}} v_{k+1} x_{j+1} v_{j} \underline{x_{j}} \quad \hat{u}
\end{aligned}
$$




$$
\begin{array}{lll}
\equiv_{\mathrm{Q}} & v_{1} x_{1} v_{2} x_{2} \ldots v_{j-2} \underline{x_{j-2}} v_{j-1} x_{j} & v_{k+1} x_{j+1} v_{j} \underline{x_{j-1}} \hat{u} \\
\equiv_{\mathrm{Q}} & \cdots & \\
\equiv_{\mathrm{Q}} & v_{1} x_{2} v_{2} x_{3} \ldots v_{j-2} x_{j-1} v_{j-1} x_{j} & v_{k+1} x_{j+1} v_{j} x_{1} \quad \hat{u}
\end{array}
$$

as asserted, where the letters in question are underlined in each step.

Step 2. Let $a \in \underline{n-1}$ and $x, y \in \underline{n}$ such that $x, y>a+1$ or $x, y<a$, then $x y$ allows the a-switch in $\mathcal{S}_{n}$.

In particular, if $v \in \mathbb{N}^{*}$ such that $\ell(v) \geq 3$, then $v$ allows the a-switch in $\mathcal{S}_{n}$.

Proof. If $x=y$, there is nothing to prove; let $x \neq y$. Let $\pi=a w(a+1) s$ such that $x y$ is a sub-word of $w$.

Consider first the case where $x, y>a+1$. We may assume that $x<y$, by Proposition 3.2. The proof is done by induction on $m:=y-x$.

If $m=1$, then $\pi \equiv \mathrm{LQ} \pi \tau_{a}$ follows from Step 1 .

Let $m>1$, and set $x_{i}:=x+i$ for all $i \in \mathbb{N}$. Inductively, the case where $x_{i}$ occurs in $s$ for all $i \in \underline{m-1}$ remains.

Choose $k \in \underline{m-1}$ maximal such that $x_{1} \pi^{-1}<x_{2} \pi^{-1}<\cdots<x_{k} \pi^{-1}$, that is

$$
\pi=a u_{1} x u_{2} y u_{3}(a+1) v_{1} x_{1} v_{2} x_{2} \ldots v_{k} x_{k} v_{k+1}
$$

for suitably chosen $u_{i}, v_{i} \in \mathbb{N}^{*}$.

If $x_{k}=y-1$, then either $m=2$ and $\pi \equiv_{\mathrm{Q}} \pi \tau_{x}$, or $m>2$ and $\pi \equiv_{\mathrm{Q}} \pi \tau_{y-1}$; in both cases, $\pi \equiv_{\mathrm{LQ}} \pi \tau_{a}$, by induction and Proposition 3.3 .

Let $x_{k}<y-1$, then $y>x_{2}$; and there is an index $j \in \underline{k}$ such that $x_{j-1} \pi^{-1}<$ $x_{k+1} \pi^{-1}<x_{j} \pi^{-1}$ (where $x_{0}:=x$ if $j=1$ ). Let $t:=a u_{1} x u_{2} y u_{3}(a+1)$, then

$$
\begin{aligned}
\pi & =t v_{1} x_{1} v_{2} x_{2} \ldots v_{j-1} x_{j-1} v_{j}^{(1)} x_{k+1} v_{j}^{(2)} x_{j} v_{j+1} x_{j+1} \ldots v_{k} x_{k} v_{k+1} \\
& \equiv_{\mathrm{Q}} t v_{1} x_{2} v_{2} x_{3} \ldots v_{j-1} x_{j} v_{j}^{(1)} x_{j+1} v_{j}^{(2)} x_{1} v_{j+1} x_{j+2} \ldots v_{k} x_{k+1} v_{k+1} \\
& =: \quad \hat{\pi}
\end{aligned}
$$

by Proposition 3.4. Furthermore, $\hat{\pi} \equiv_{\mathrm{Q}} \hat{\pi} \tau_{x}$, since $x \hat{\pi}^{-1}<x_{2} \hat{\pi}^{-1}<x_{1} \hat{\pi}^{-1}$. By induction, $\hat{\pi} \tau_{x} \equiv \mathrm{LQ}\left(\hat{\pi} \tau_{x}\right) \tau_{a}$, hence also $\hat{\pi} \equiv \mathrm{LQ} \hat{\pi} \tau_{a}$, by Proposition 3.3. Another application of Proposition 3.3 yields $\pi \equiv_{\mathrm{LQ}} \pi \tau_{a}$ and completes the proof in the case $x, y>a+1$. 
Now assume that $x, y<a$, then $n+1-x, n+1-y>(n-a)+1$, hence $(n+1-x)(n+1-y)$ allows the $(n-a)$-switch in $\mathcal{S}_{n}$, by the part already proven. As a consequence, $x y$ allows the $a$-switch in $\mathcal{S}_{n}$, by Proposition 3.2 . If $v \in \mathbb{N}^{*}$ such that there are three distinct letters $\neq a, a+1$ occurring in $v$, then at least two of these are $<a$ or $>a+1$. This completes the proof.

Step 3. Let $a \in \underline{n-1}$ and $v \in \mathbb{N}^{*}$ such that $\ell(v) \geq 2$, then $v$ allows the a-switch in $\mathcal{S}_{n}$.

Proof. By (3) and Step 2, the case where $v=x y$ such that and $x<a-1$ and $y>a+2$, or $y<a-1$ and $x>a+2$, remains. By Proposition 3.2, it suffices to consider the case of $x<a-1$ and $y>a+2$. Let $\pi=a w(a+1) s \in \mathcal{S}_{n}$ such that $x y$ is a sub-word of $w$. If $\ell(w) \geq 3$, then $\pi \equiv_{\mathrm{LQ}} \pi \tau_{a}$ follows from Step 2 .

Let $w=x y$, then each of the letters $x+1, x+2, \ldots, a-1$ occurs in $s$, and $y>a+1>a>a-1 \geq x+1$. Applying Step a number of times implies

$$
\pi \equiv \mathrm{LQ} \pi \tau_{x} \equiv \mathrm{LQ} \pi \tau_{x} \tau_{x+1} \equiv \mathrm{LQ} \cdots \equiv_{\mathrm{LQ}} \pi \tau_{x} \tau_{x+1} \cdots \tau_{a-2}=a(a-1) y(a+1) \hat{s}
$$

for a properly chosen $\hat{s} \in \mathbb{N}^{*}$, hence $\pi \equiv \mathrm{LQ} \pi \tau_{a}$ as asserted, by Proposition 3.3 .

As the final step, we are now in a position to state and prove

Theorem 2. Lie $\cap \mathcal{Q} \subseteq \mathcal{D}$.

Proof. It suffices to prove $\operatorname{Lie}_{n} \cap \mathcal{Q}_{n} \subseteq \mathcal{D}_{n}$, since Lie $\cap \mathcal{Q}=\bigoplus_{n \geq 0} \operatorname{Lie}_{n} \cap \mathcal{Q}_{n}$. By Proposition 3.1, Step 3 and (6), it thus remains to be shown that $\pi \equiv \mathrm{LQ} \pi \tau_{a}$ whenever $x, a \in \underline{n}, s \in \mathbb{N}^{*}$ such that $\pi=a x(a+1) s \in \mathcal{S}_{n}$.

If $n=3$, this is immediate. Let $n>3$ and choose $y \in \mathbb{N}$ and $u \in \mathbb{N}^{*}$ such that $s=y u$, then

$$
\begin{aligned}
& \pi=a x(a+1) y u \\
& =a((a+1) ш x y u)-a(a+1) x y u-a x y((a+1) ш u) \\
& \equiv_{\mathrm{L}} \quad-(a+1) a x y u-a(a+1) x y u-a x y((a+1) \omega u) \text {, by (目) } \\
& \equiv_{\mathrm{LQ}}-(a+1) a x y u-a(a+1) x y u-(a+1) x y(a ш u) \text {, by Step } 3 \\
& \equiv_{\mathrm{L}} \quad(a+1)(a ш x y u)-(a+1) a x y u-(a+1) x y(a ш u), \text { by (蛋) } \\
& =(a+1) x a y u \\
& =\pi \tau_{a} \text {. }
\end{aligned}
$$

The theorem is proved. 
Denote by $\Delta$ the coproduct in $\mathcal{P}$ (as in [MR95, p. 977]), and by $\emptyset$ the unique element in $\mathcal{S}_{0}$. For any a Hopf sub-algebra $\mathcal{A}$ of $\mathcal{P}$, let

$$
\operatorname{Prim}(\mathcal{A})=\{\alpha \in \mathcal{A} \mid \Delta(\alpha)=\alpha \otimes \emptyset+\emptyset \otimes \alpha\}
$$

be the primitive Lie algebra of $\mathcal{A}$, and denote by $\operatorname{Prim}(\mathcal{A})_{n}$ its $n$-th homogeneous component. The sub-algebra $\mathcal{C}$ of $\mathcal{P}$ generated by $\operatorname{Prim}(\mathcal{P})$ (the domain of co-commutativity of $\mathcal{P}$ ) contains $\mathcal{L}$. Furthermore, $\mathcal{C} \cap \mathcal{Q}$ (the domain of co-commutativity of $\mathcal{Q}$ ) is generated by $\operatorname{Prim}(\mathcal{Q})$ and contains $\mathcal{D}=\mathcal{L} \cap \mathcal{Q}$.

It turns out that $\mathcal{D}$ is strictly contained in $\mathcal{C} \cap \mathcal{Q}$. Indeed, $\varphi=3412+2413-$ $(3142+2143) \in \mathcal{Q}_{4} \backslash \mathcal{D}_{4}$, and $\Delta(\varphi)=\varphi \otimes \emptyset+\emptyset \otimes \varphi$. For $n=4,5,6$, the dimension of $\operatorname{Prim}(\mathcal{D})_{n}$ is, respectively, 3,6, and 9, while the dimension of $\operatorname{Prim}(\mathcal{Q})_{n}$ is, respectively, 4, 9, and 26. A description of the elements of $\operatorname{Prim}(\mathcal{D})_{n}$ as well as of its dimension is known in general ([BL93, 4.5], [BL96, $1.5])$. It would be of interest if analogous results for $\mathcal{Q}$ were obtained.

\section{References}

[AS] M. Aguiar and F. Sottile. Structure of the Malvenuto-Reutenauer Hopf algebra of permutations. Preprint math.CO/020328228.

[BL93] D. Blessenohl and H. Laue. Algebraic combinatorics related to the free Lie algebra. In Publ. I.R.M.A Strasbourg, Actes $29^{\mathrm{e}}$ Séminaire Lotharingien, pages 1-21, 1993.

[BL96] D. Blessenohl and H. Laue. On the descending Loewy series of Solomon's descent algebra. J. Algebra, 180:698-724, 1996.

[BS] D. Blessenohl and M. Schocker. Noncommutative character theory of symmetric groups I: the Jöllenbeck method. Preprint.

[Cha00] F. Chapoton. Bigèbres différentielles graduées associées aux permutoèdres, associaèdres et hypercubes. Ann. Inst. Fourier (Grenoble), 50:1127-1153, 2000.

[DHT] G. Duchamp, F. Hivert, and J.-Y. Thibon. Noncommutative symmetric functions VI: Free quasi-symmetric functions and related algebras. Preprint.

[Duc91] G. Duchamp. Orthogonal projection onto the free Lie algebra. Theoret. Comput. Sci, 79:227-239, 1991. 
[Dyn47] E. B. Dynkin. Calculation of the coefficients of the CampbellHausdorff formula. Docl. Akad. Nauk SSSR (N. S.), 57:323-326, 1947.

[GKL $\left.{ }^{+} 95\right]$ I. M. Gelfand, D. Krob, A. Lascoux, B. Leclerc, V. Retakh, and J.-Y. Thibon. Noncommutative symmetric functions. Adv. in Math., 112(2):218-348, 1995.

[GR89] A. M. Garsia and C. Reutenauer. A decomposition of Solomon's descent algebra. Adv. in Math., 77:189-262, 1989.

[Jö199] A. Jöllenbeck. Nichtkommutative Charaktertheorie der symmetrischen Gruppen. Bayreuth. Math. Schr., 56:1-41, 1999.

[JR01] A. Jöllenbeck and C. Reutenauer. Eine Symmetrieeigenschaft von Solomons Algebra und der höheren Lie-Charaktere. Abh. Math. Sem. Univ. Hamburg, 71:105-111, 2001.

[Knu70] D. E. Knuth. Permutations, matrices and generalized Youngtableaux. Pacific J. Math., 34:709-727, 1970.

[LR98] J.-L. Loday and M. O. Ronco. Hopf algebra of the planar binary trees. Adv. in Math., 139:293-309, 1998.

[LS81] A. Lascoux and M. P. Schützenberger. Le monoïde plaxique. In Noncommutative Structures in algebra and geometric combinatorics, A. de Luca Ed., Quaderni della Ricerca Scientifica del C.N.R., Roma, 1981.

[MM65] J. W. Milnor and J. C. Moore. On the structure of Hopf algebras. Ann. of Math., 81:211-264, 1965.

[MR95] C. Malvenuto and C. Reutenauer. Duality between quasi-symmetric functions and the Solomon descent algebra. J. Algebra, 177:967-982, 1995.

[PR95] S. Poirier and C. Reutenauer. Algébres de Hopf de Tableaux. Ann. Sci. Math. Québec, 19(1):79-90, 1995.

[PR01] F. Patras and C. Reutenauer. Lie representations and an algebra containing Solomon's. Preprint, 2001.

[Reu93] C. Reutenauer. Free Lie Algebras, volume 7 of London Mathematical Society monographs, new series. Oxford University Press, 1993. 
[Sch61] C. Schensted. Longest Increasing and Decreasing Subsequences. Canad. J. Math., 13:179-191, 1961.

[Sch63] M. P. Schützenberger. Quelques remarques sur une construction de Schensted. Math. Scand., 12:117-128, 1963.

[Sol76] L. Solomon. A Mackey formula in the group ring of a Coxeter group. J. Algebra, 41:255-268, 1976.

[Spe48] W. Specht. Die linearen Beziehungen zwischen höheren Kommutatoren. Mathematische Zeitschrift, 51:367-376, 1948.

[Wev49] F. Wever. Über Invarianten in Lieschen Ringen. Mathematische Annalen, 120:563-580, 1949. 